\title{
O ESTUDO DOS MÉTODOS ESTATÍSTICOS NA CONTABILIDADE GERENCIAL POR MEIO DA BIBLIOMETRIA ATRAVÉS DA LEI DE LOKTA
}

THE STUDY OF STATISTICAL METHODS IN MANAGERIAL ACCOUNTING THROUGH BIBLIOMETRY THROUGH LOKTA'S LAW

MARILÉA DA SILVA OLIVEIRA

Universidade do Estado do Rio de Janeiro

ROBERTA DA SILVA LOPES

Universidade do Estado do Rio de Janeiro

GUILHERME TEIXEIRA PORTUGAL

Universidade do Estado do Rio de Janeiro JOSÉ FRANCISCO MOREIRA PESSANHA

Universidade do Estado do Rio de Janeiro

Recebido em 25/11/18

Avaliado pelo sistema double blind review

Aceito para publicação pelo Editor Chefe Dr. Leonardo José Seixas Pinto em 25/12/18 e publicado em 28/12/18

\section{RESUMO}

O estudo tem como objetivo mapear a utilização dos métodos estatísticos em contabilidade gerencial no período de 2013 a 2016. A fim de atingir o objetivo proposto, realizou-se uma pesquisa quantitativa, exploratória, descritiva e bibliométrica baseada na Lei de Lokta, importante para se avançar nos conhecimentos científicos dos métodos estatísticos utilizados em contabilidade gerencial. A coleta de dados foi através da seleção de artigos na base de dados SPELL no quadriênio com classificação A2, B1 e B2, realizada pela Coordenação de Aperfeiçoamento de Pessoal de Nível Superior - Capes. Os resultados alcançados mostram que há correlação dos artigos publicados por autor (artigos com aplicações de métodos estatísticos) com o estimado pela Lei de Lokta. Em comparação com o modelo de Poisson a produtividade informada pela quantidade de trabalho por autor estimada nesse modelo está de encontro à produtividade pela Lei de Lokta. Esses resultados em epígrafe poderão ser uteis para estimular futuras pesquisas empíricas mais aprofundadas ao auxiliar pesquisadores e professores com objetivos específicos de determinar aplicabilidade da estatística em Contabilidade Gerencial.

Palavras-chaves: Métodos Estatísticos; Contabilidade Gerencial; Bibliometria; Lei de Lokta.

\section{ABSTRACT}

The aim of this study is to map the use of statistical methods in managerial accounting in the period from 2013 to 2016. In order to achieve the proposed objective, a quantitative, exploratory, descriptive and bibliometric study was carried out based on the Lokta Law, important for to advance the scientific knowledge of statistical methods used in managerial accounting. The data collection was through the selection of articles in the SPELL database in the four - year period with classification A2, B1 and B2, carried out by the Coordination of Improvement of Higher - Level Personnel - Capes. The results show that there is a correlation between articles published by author (articles with applications of statistical methods) and those estimated by Lokta 's Law. In comparison with the Poisson model, the productivity reported by the amount of work per author estimated in this model is against productivity by Lokta's Law. These results may be useful to stimulate further empirical research in order to assist researchers and professors with specific objectives to determine the applicability of statistics in Managerial Accounting.

Keywords: Statistical methods; Management accounting; Bibliometric; Law of Lokta. 


\section{INTRODUÇÃO}

A estatística tem sido utilizada em diversas abordagens da contabilidade gerencial como ferramenta de gestão e tratamentos da informação contábil. Alguns estudiosos (Corrar \& Theóphilo, 2015) já utilizam os métodos estatísticos, bem como a matemática e a informática aplicados à contabilidade gerencial como uma nova modalidade de gestão e tratamento da informação chamada de contabilometria. M. C. DaSilva, Chacon, e J. dos Santos (2005, p. 3) afirmam que a "Contabilometria pode ser considerada como uma metodologia científica fundamentada nos Métodos Quantitativos (Matemática, Estatística e Informática) para uso na Contabilidade com o objetivo de auxiliar o Gestor na tomada de decisões".

O uso da estatística é importante para estudos e aplicabilidades futuras nos temas abordados em contabilidade gerencial, como forma de reduzir a subjetividade, melhoria da transparência e qualidade da informação contábil (Wernke e Bornia, 2001). Sérgio Aparecido Ignácio (2010, p.1) destaca que "A estatística na atualidade tem contribuído de forma significativa para o processo de tomada de decisão . . . Onde houver incerteza, essa ferramenta pode ser usada."

Iudícibus (1982) publicou o primeiro artigo intitulado "Existirá a contabilometria?", em que já se questionava a futura existência dessa metodologia científica. Mais recentemente Dallanona, Nascimento e Hein em 2010 pesquisam, através da bibliometria, os métodos estatísticos usados nas dissertações de trabalhos de pós-graduação na área de Ciências Contábeis.

O presente artigo foi motivado pelo interesse em mapear a utilização dos métodos estatísticos em contabilidade gerencial no período de 2013 a 2016. O mapeamento por meio da bibliometria auxilia no entendimento do Gap que existe entre uso dos métodos estatísticos e aplicabilidade em Contabilidade Gerencial. E, por meio dessa análise pretende-se responder a seguinte questão: Dentre os artigos em Contabilidade Gerencial, quais utilizam os métodos estatísticos? O presente trabalho busca contribuir com a informação de quais são os métodos estatísticos mais usados na produção científica em Contabilidade Gerencial, por meio do mapeamento bibliométrico dos artigos científicos que utilizaram os métodos estatísticos como instrumento de análise e de pesquisa. Além de estimular futuras pesquisas empíricas mais aprofundadas ao auxiliar pesquisadores e professores com objetivos específicos de determinar aplicabilidade da estatística em Contabilidade Gerencial.

\section{FUNDAMENTAÇÃO TEÓRICA}

Para o entendimento da revisão da literatura esta seção está dividida em duas subseções. A primeira tem por objetivo apresentar a evolução bibliográfica da contabilidade gerencial, da sua importância na atualidade e da aplicabilidade com o uso dos métodos estatísticos. A segunda, apresenta a abordagem conceitual dos métodos estatísticos.

\subsection{Ferramentas Quantitativas na Contabilidade Gerencial: Uma Abordagem Bibliográfica Evolutiva}

O International Federation of Accountants (IFAC) (1998, como citado em Ribeiro \& Espejo, 2013), no pronunciamento conhecido por International Management Accounting Practice 1 (IMAP 1) define a evolução da contabilidade gerencial em quatro estágios. Dentre esses estágios, o quarto estágio (a partir de 1985 até atualidade) destacam-se no uso do Balanced Scorecard (BSC), Gestão Econômica (GECON) e Economic Value Added (EVA), dentre outros. Além disso, na fase atual, os métodos estatísticos estão presentes em diversos setores aplicáveis à Contabilidade Gerencial, como se vê de acordo com a Tabela 1 evolutiva. 
Tabela 1: Abordagem bibliográfica evolutiva da Contabilidade Gerencial

Período Autor Características principais

1982 IUDICÍBUS

1999

2000

FIGUEREDO e MOURA

2001 WERNKE e BORNIA

2005

2006

BOENTE, MELO, TARSO e ARAUJO

DIEHL, SOUZA

e DOMINGOS

2010 SILVA, PONTES $\mathrm{P}$ VILLELA
No início da década de 80, no Brasil a pesquisa sobre métodos estatísticos, matemática, informática aplicados às soluções de problemas contábeis reais foi intitulado por Contabilometria. (Iudícibus, 1982). Para o referido autor (Iudícibus, 1982, p.41) a "Contabilometria seria: . . . a análise quantitativa de "fenômenos contábeis" reais baseada no desenvolvimento concomitante da teoria e da observação, relacionados através de métodos apropriados de inferência ...”.

A contabilidade gerencial "será eficaz se contiver um sistema de informações contábeis integrados ao sistema de gestão empresarial, que é aquele que reúne e consolida todas as informações relevantes e necessárias para gerir a organização". Além disso, o referido autor destaca a importância de "se considerar o controle como uma forma de se estudar novas possibilidades ou analisar novos caminhos que possibilitem redução de custos, agilidade de operação, maximização de lucros e manutenção da qualidade do produto ou serviço prestado pela empresa." (Coelho, 1999, p.26).

No artigo, "A Utilização dos Métodos Quantitativos pela Contabilidade para Otimização de Receitas e Racionalização de Custos", os autores mencionam a aplicabilidade de valor esperado e desvio padrão, assim como a média aritmética das probabilidades para subsidiar a decisão de investimento de capital de longo prazo.

Os autores afirmam que os métodos multicritérios atribuem valor à informação contábil por permitir a abordagem de problemas considerados complexos e, portanto, não tratáveis pelos procedimentos intuitivo-empíricos usuais, assim como, por conferir "ao processo de tomada de decisão uma clareza e conseqüente transparência não disponíveis quando esses procedimentos, ou outros métodos de natureza monocritério, são utilizados.” (Wernke \& Bornia, 2001, p.1).

Menciona que uma das técnicas de métodos quantitativos é a regressão linear, a qual é "usada para melhorar a capacidade de analisar o comportamento dos custos e o aumento da objetividade e confiabilidade das informações contábeis, através da análise individual das variáveis que possam estar relacionadas com a composição do custo." (Sell, 2005, p.1).

Boente, Melo, Tarso e Araujo (2006) realizam um estudo de caso em uma empresa comercial utilizando os métodos quantitativos pela necessidade de se avaliar o impacto dos custos a partir da variação do nível de atividade, de segurança e de agilidade, normalmente, proporcionadas por metodologias estatísticas.

Menciona o uso da estatística descritiva na pesquisa de custo, através de estudo bibliométrico em que o uso desse método foi mais presente na controladoria. "A área temática que mais fez uso da estatística descritiva, em números absolutos, foi a Controladoria, área temática 15. Dos 49 artigos aprovados, 37 se serviram da estatística descritiva." (Diehl, M. A. DeSouza, \& Domingos, 2007, p.12).

A. M. H. L. Silva, Pontes e Villela (2010, p.1) evidenciam que com "a globalização e a constante modernização empresarial, a contabilidade gerencial precisa estar apta a acompanhar esses avanços e continuar analisando e fornecendo as informações necessárias às tomadas de decisões das empresas." Ainda de acordo com os autores, a contabilidade gerencial pode usufruir da mineração de dados, que é um exemplo dessa tecnologia da modernização. A mineração de dados “.... tem por objetivo principal descobrir informações relacionadas e concisas, às vezes, desconhecidas até pela própria empresa, em meio a 
Continua...

SILVA, WANDERLEY e SANTOS

2011

2013

2014

2017 suas enormes bases de dados, pois, na maioria das vezes, esses dados estão espalhados e não vinculados da maneira correta." (A. M. H. L. Silva et al., 2010, p.1).

Fávero L L (2009, como citado em G. I. S. DaSilva, Wanderley, \& R. S. Santos, 2011, p.15) salienta que a análise multivariada tem apresentado uma importante função na tomada de decisões em diversas áreas do saber, tais como as áreas de economia, administração, finanças ou contabilidade, as quais possuem uma vasta quantidade e diversidade de dados, que por sua vez dificulta "a determinação do relacionamento entre as variáveis e, principalmente, do modelo mais apropriado para se chegar às respostas desejadas."

Rezende e Carmo (2013, p.3) declara "promover a utilização de REZENDE e métodos quantitativos como meio para ampliar a capacidade CARMO informativa e gerencial daquela metodologia de custeamento, no que tange às análises do tipo custo-volume-lucro, doravante denominada apenas de análises do tipo CVL."

$\mathrm{O}$ autor afirma que "os métodos quantitativos, principalmente as suas fundamentações matemáticas e estatísticas, são instrumentos imprescindíveis na contabilidade e na gestão." (Pereira, 2014, p.23). E, ainda, que a programação linear pode ser usada nas ciências sociais, inclusive na contabilidade, a fim de gerar várias informações importantes para as organizações na tomada de decisão. A programação linear pode ser usada, por exemplo, para dimensionar investimentos, maximizar os lucros e minimizar os custos. (Pereira, 2014, p.198).

S. A. L. Silva, Sant'Ana, Menezes, Paula e Chain (2017, p.38) destacam a importância do uso de recursos eletrônicos em contabilidade e finanças, tal uso ". . . permitiu uma mudança no perfil das pesquisas científicas na área, já que ao longo dos últimos 50 anos, com a adoção dos fundamentos do funcionalismo positivista, os cientistas passaram a utilizar predominantemente métodos e técnicas quantitativas, em uma filosofia normativa." Os autores (2017) salientam ainda, que existe uma mudança de perfil do pesquisador, assim como é observado um fator limitante, que é a baixa oferta nas grades curriculares de disciplinas com enfoque quantitativo, nos cursos de graduação em ciências contábeis oferecidas pelas Universidades Federais no país. "Desse fato decorre a falta de contato desses acadêmicos com softwares estatísticos. Ocorre que para esta alteração de perfil científico, em um ambiente que exige predominantemente a utilização da estatística e matemática, a aplicação de softwares estatísticos tornou-se indispensável.” (S. A. L. Silva et al., 2017, p.38).

Fonte: Elaborado pela autora (2017)

Nesse sentido, Crepaldi (2008, p.5) afirma que a Contabilidade Gerencial é "o ramo da contabilidade que tem por objetivo fornecer instrumentos aos administradores de empresas que os auxiliem em suas funções gerenciais." Sendo assim na Contabilidade Gerencial:

... percebemos a importância da estatística. Além disso, se considerarmos que a função da contabilidade é disponibilizar aos usuários do sistema contábil uma gama de informações ajustadas e convenientes ao processo decisório estratégico das organizações, é fácil entender por que a utilização de modelos contábeis com base em métodos quantitativos tem se tornado uma ferramenta cada vez mais solicitada-também em decorrência do desenvolvimento da tecnologia da informação. (Pereira, 2014, p.17).

Portanto, a Contabilidade Gerencial embora seja considerada um "alimentador das informações utilizadas pelos gestores empresariais" conforme destaca De Souza, Lisboa, e Rocha 
(2003, p. 41) ainda é necessário mais pesquisas com intuito de avançar nessa fronteira do conhecimento.

\subsection{Abordagem Conceitual dos Métodos Estatísticos}

De acordo com o Ignácio (2010), a utilização dos métodos estatísticos tem grande importância na contribuição à contabilidade. $\mathrm{O}$ autor destaca ainda que:

A estatística na atualidade tem contribuído de forma significativa . .., pois grande parte do que se faz se baseia em métodos quantitativos, e a estatística é uma dessas áreas. Na era da informação e do conhecimento, a estatística utiliza a matemática para dar apoio aos profissionais da iniciativa privada, do governo e pesquisadores. (Ignácio, 2010, p.1).

B. M. Santos, Lemes, W. S. Silva, e Alves (2014) mencionam que os principais instrumentos estatísticos estão no ambiente empresarial como ferramenta e parâmetro de análise para o gestor. Além disso, o autor destaca também que "em uma empresa é possível utilizar do método de distribuição de frequências para analisar cada recurso de acordo com sua quantidade e classe ..." (B. M. Santos, Lemes, W. S. Silva, \& Alves , 2014, p.3).

Figueiredo e Moura (2001) afirmam que o uso das ferramentas estatísticas na Contabilidade, através dos métodos quantitativos aplicados e o avanço da tecnologia da informação estão cada vez mais recorrentes. Esses autores mencionam que "a utilização de modelos contábeis baseados em métodos quantitativos tem se tornado cada vez mais frequente decorrente do rápido desenvolvimento da tecnologia da informação e da utilização corriqueira dos microcomputadores." (Figueredo \& Moura, 2001, p.1).

Nesse contexto, Dallanona, Nascimento e Hein (2010, p. 60) classificaram os principais métodos estatísticos, em Estatística descritiva, Inferências estatísticas, Regressão e Correlação, Análise Multivariada de dados, Abordagem multicritério, Modelagem Matemática, Metaheurística e Data mining presentes na literatura à luz dos autores que tratam sobre o tema como Spiegel (1993), Maroco (2003), L. J. Corrar, Paulo e Dias Filho (2007), e V. DosSantos, R. C. Klann, R. B. Rausch (2010), na Tabela 2 especificada.

Tabela 2: Principais Métodos Estatísticos

\begin{tabular}{ll}
\hline $\begin{array}{l}\text { Métodos } \\
\text { estatísticos }\end{array}$ & \multicolumn{1}{c}{ Descrição } \\
\hline $\begin{array}{l}\text { Estatística } \\
\text { descritiva }\end{array}$ & $\begin{array}{l}\text { Fornecem descrições quantitativas de uma variável aleatória, por exemplo, distribuições de } \\
\text { frequência, medidas de tendência central (média, mediana e moda), medidas de dispersão } \\
\text { (amplitude, desvio-padrão e coeficiente de variação), bem como quantis e medidas de assimetria } \\
\text { e achatamento. }\end{array}$
\end{tabular}

Inferências estatísticas

Permitem deduzir propriedades para uma população inteira a partir da análise de uma amostra aleatória de dados, extraída da população investigada. Envolve o dimensionamento e o desenho do processo de extração da amostra, a estimação dos parâmetros de interesse a partir dos dados amostrais e testes de significância estatística. $\begin{array}{ll}\text { Correlação } & \text { relaciona o valor esperado } \mathrm{E}(\mathrm{Y}) \text { da variável resposta } \mathrm{Y} \text { com os valores dados de variáveis }\end{array}$ explicativas $X_{1}, \ldots, X_{p}$.

Análise Abrange um amplo conjunto de métodos, desde os métodos para análise da dependência entre Multivariada variáveis (modelos de regressão linear e modelos lineares generalizados) até modelos de de dados interdependência entre variáveis, por exemplo: análise de componentes principais, análise fatorial, análise fatorial de correspondência, análise discriminante, análise de agrupamentos e escalonamento multidimensional.

Abordagem Análise envoltória de dados (DEA), análise hierárquica de processos (HPA). multicritério

Modelagem Matemática

Resolução de problemas de otimização por meio de modelos de Programação Matemática, por exemplo, Programação linear e Programação Não linear. 
As próximas seções apresentam os enquadramentos metodológicos, descrevendo a metodologia, população e amostra e, sobre a bibliometria, os procedimentos para a coleta e tratamento dos dados, posteriormente a análise dos dados e resultados e considerações finais.

\section{ENQUADRAMENTO METODOLÓGICO}

A metodologia utilizada neste trabalho tem natureza aplicada, com objetivos exploratório e descritivo, com procedimentos bibliográficos, comparativos, estatísticos e bibliométricos, baseada na classificação determinada por Lakatos e Marconi (2002), Prodanov e Freitas (2013).

No procedimento comparativo, foram comparados os artigos que utilizavam os métodos estatísticos e os que não utilizavam, conforme mencionado por Prodanov e Freitas (2013, p.38) o método comparativo utiliza as explicações dos fenômenos por meios de análises dos dados concretos e ajuda na dedução dos elementos abstratos, constantes ou gerais.

\subsection{Bibliometria}

Quanto às Leis bibliométricas, existem três, conforme J. E. R. Monteiro et al. (2011, p. 7), conforme ilustra a Tabela 3 .

Tabela 3: Leis bibliométricas

\begin{tabular}{|c|c|}
\hline LEIS BIBLIOMÉTRICAS & CARACTERÍSTICAS \\
\hline $\begin{array}{l}\text { Lei de Lokta }{ }^{\mathrm{a}} \\
\propto_{\mathrm{n}}=\propto_{1} \cdot\left(1 / \mathrm{n}^{\mathrm{c}}\right)\end{array}$ & $\begin{array}{l}\text { Conhecida como Lei do quadrado inverso, que indica a } \\
\text { quantidade da produtividade dos pesquisadores, por meio } \\
\text { de modelos distribuídos em tamanho e frequência, dos } \\
\text { vários pesquisadores em um conjunto de publicações. }\end{array}$ \\
\hline $\begin{array}{l}\text { Lei de Bradford } \\
F(x)=a+b \log x\end{array}$ & $\begin{array}{l}\text { Conhecida como Lei do mínimo esforço, pois se } \\
\text { fundamenta na verificação do número de ocorrência ou } \\
\text { frequência que determinadas palavras aparecem em um } \\
\text { conjunto de textos. } \\
\text { Conhecida como Lei da dispersão, pois mede a } \\
\text { produtividade das revistas, estabelecendo o núcleo e as } \\
\text { áreas de dispersão a respeito de um assunto escolhido num } \\
\text { conjunto de revistas. }\end{array}$ \\
\hline
\end{tabular}

Fonte: Adaptado dos autores J. E. R. Monteiro et al. (2011)

${ }^{a}$ Nota: $\propto_{\mathrm{n}}$ (número de autores que publicam um n-artigos); $\propto_{1}$ (número de autores que publicam um único artigo) e $\mathrm{n}$ (número de artigos).

${ }^{\mathrm{b}}$ Nota: $\mathrm{f}=\mathrm{b} / \mathrm{r}$.a, onde $\mathrm{f}$ (frequência de uma palavra na posição $\mathrm{r}$ ), b e a (são valores constantes, com a variável a próximo do número 1).

${ }^{\mathrm{c}}$ Nota: $\mathrm{F}(\mathrm{x})$ (número cumulativo de referências encontradas no x que são números de periódicos de maiores produções e, a e b são coeficientes).

Urbizagastegui (2008, p. 90) informa que existem três maneiras de contagem para a Lei de Lokta que são contagem direta, contagem completa e contagem ajustada. A primeira maneira é feita a contagem da contribuição somente com os autores principais, desconsiderando o(s) coautor(es). $\mathrm{Na}$ contagem completa é considerada tanto autor quanto coautor(es). E, na ajustada é contada com uma fração ou porção da contribuição total do(s) autor(es) principal (ais) e/ou secundário. Nessa pesquisa, foi adotada a contagem completa.

\subsection{Procedimentos Para Coleta e Tratamento dos Dados}

Nesta seção está subdividida em duas subseções. A primeira aborda sobre a seleção dos artigos a partir da pesquisa bibliográfica. E, a segunda menciona como os dados foram tratados.

\subsubsection{Seleção dos Artigos na Pesquisa Bibliográfica}

No exame dos dados foi utilizada a técnica qualitativa, a fim de analisar o conteúdo, com uma leitura comum e criteriosa de cada artigo identificado na busca e com revisão detalhada das produções científicas que apresentaram a citação efetuada em assuntos relacionados à contabilidade gerencial. 
Já a pesquisa e análise bibliográfica foram utilizadas como técnicas de coleta de dados, que buscou definir quais autores utilizam a estatística em suas produções científicas envolvendo a contabilidade gerencial.

Nessa pesquisa foi necessária a definição da palavra-chave "contabilidade gerencial" como forma de seleção das produções científicas. Na coleta de dados, foi feito uma seleção de artigos em maio de 2017 na base de dados SPELL no último quadriênio com classificação A2, B1 e B2, realizada pela Coordenação de Aperfeiçoamento de Pessoal de Nível Superior - Capes.

Para identificar esses artigos foram estabelecidos os seguintes procedimentos: (i) digitação da terminologia "contabilidade gerencial" na pesquisa avançada; (ii) relacionados somente artigos na área de conhecimento de contabilidade no idioma português; (iii) intervalo temporal de 2013 até 2016.

No processo de busca no período citado retornou um total de 70 artigos nacionais. Desses artigos coletados, somente 51 se remetiam à contabilidade gerencial ou temas relacionados à contabilidade gerencial, os quais se enquadraram nos critérios de busca. A outra metade dos artigos coletados nessa base, nenhum se enquadrou no quesito proposto neste estudo, portanto, não foram considerados para esse estudo específico. Desses 51 artigos, foram classificados os que empregam técnicas estatísticas e os que não aplicam técnicas estatísticas nas análises dos temas abordados em contabilidade gerencial, conforme demonstrado na Figura 1.

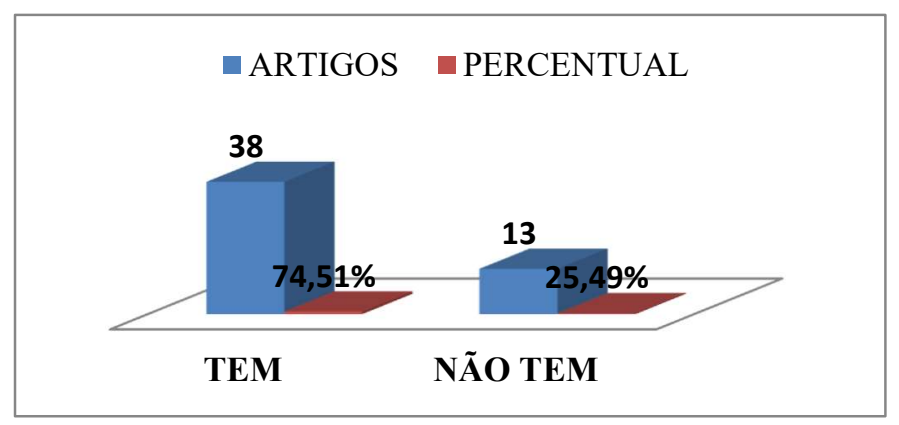

Figura 1: Percentual do total de artigos que tem e não tem estatística Fonte: dados da pesquisa, 2017

\subsubsection{Tratamento dos dados}

Para verificar a duplicidade dos artigos foi realizada uma análise bibliográfica através do software Mendeley, o qual não apresentou duplicidade nos artigos analisados.

Após a análise de conteúdo os artigos foram organizados na seguinte ordem de detalhamento: título do artigo, ano da publicação, nome do autor, autora, autores ou autoras, Qualis Capes, nome da revista, IES (Instituições de Ensino Superior), quais as técnicas estatísticas foram aplicadas e a classificação que informa se o foco do estudo é contabilidade gerencial ou não.

Para aferição dos produtores de artigos, a fim de observar à Lei de Lokta, foi realizada a opção pelo sistema de contagem completa, o qual os autores primários e secundários de uma só produção científica foram atribuídos como contribuintes a essa produção.

De acordo com Urbizagastegui (2008, p. 100) é possível analisar modelo probabilístico do Poisson Lognormal a produtividade da Lei de Lokta. Com isso para a mensuração dos dados foi considerado também o modelo estatístico probabilístico Poisson Lognormal para comparabilidade com o Lokta.

\section{ANÁLISE DOS DADOS E RESULTADOS}

\section{i) Lei de Lokta Para o Total de Artigos Pesquisados na Base Spell}

$\mathrm{Na}$ Tabela 4 apresenta-se a produtividade dos autores segundo o critério da contagem completa no total dos artigos pesquisados na base Spell, relacionando os autores com uma só produção científica, com aqueles que possuem uma frequência de dois, quatro, cinco e nove artigos publicados, após a análise realizada desses autores nas publicações em revistas A2, B1 e B2. 
Tabela 4: Lei de Lokta para os artigos pesquisados

\begin{tabular}{cccc}
\hline $\begin{array}{c}\text { Artigos } \\
\text { por autor }\end{array}$ & $\begin{array}{c}\text { Número de } \\
\text { autores } \\
\text { absoluto }\end{array}$ & $\begin{array}{c}\text { \% de autores } \\
\text { absolutos }\end{array}$ & $\begin{array}{c}\text { Padrão } \\
\text { Lokta }\end{array}$ \\
\hline 1 & 145 & $90 \%$ & $60,8 \%$ \\
\hline 2 & 11 & $7 \%$ & $15,20 \%$ \\
\hline 4 & 2 & $1 \%$ & $3,80 \%$ \\
\hline 5 & 2 & $1 \%$ & $2,43 \%$ \\
\hline 9 & 1 & $1 \%$ & $0,75 \%$ \\
\hline TOTAL & $\mathbf{1 6 1}$ & $\mathbf{1}$ & $\mathbf{8 3 \%}$ \\
\hline \multicolumn{5}{c}{ Fonte: dados da pesquisa, 2017. } \\
\hline
\end{tabular}

Pela análise da produtividade dos autores por meio da forma de contagem completa, segundo a Tabela 4, foram observados 161 autores, sendo 90\% produziram somente um artigo. Em comparação com o padrão da Lei de Lokta de 60,8\%, a produtividade desses autores superou em 29,2 pontos percentuais, atendendo os pressupostos dessa lei em que a maioria dos autores publica somente um artigo e poucos autores com produções mais relevantes.

Do total dos artigos classificados como Contabilidade Gerencial na busca avançada, os autores que se destacaram possuem entre 4 e 9 artigos.

Em observação da Figura 2 abaixo, que retrata a produtividade percentual do total dos autores com o padrão Lei de Lokta, mostra que a tendência dos números de autores com artigos publicados na ordem de um a nove artigos por autor, diminuem, formando um $\mathrm{J}$ conforme preconizado pela literatura e ratificado pelo Urbizagastegui (2008), base para esse estudo.

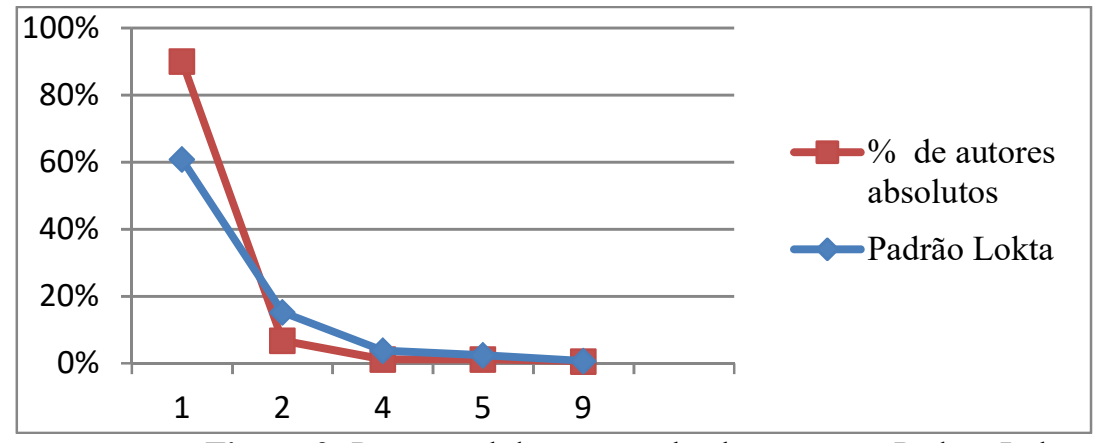

Figura 2: Percentual de autores absolutos versus Padrão Lokta Fonte: dados da pesquisa, 2017

\section{ii) Aplicação dos Métodos Estatísticos em Contabilidade Gerencial}

Dos 51 artigos classificados como contabilidade gerencial, somente 38 utilizaram métodos estatísticos nas abordagens dos temas em contabilidade gerencial para os estudos pesquisados. Desse total que utilizaram métodos estatísticos todos apresentaram a estatística descritiva como técnica utilizada inicial para análise das pesquisas, como demonstrado na Tabela 5.

Tabela 5:Métodos estatísticos aplicados

\begin{tabular}{c|c|c}
\hline $\begin{array}{c}\text { Métodos Estatísticos } \\
\text { aplicados nos estudos } \\
\text { analisados }\end{array}$ & Artigos & Percentual \\
Estatística descritiva & 38 & $70 \%$ \\
Inferências estatísticas & 7 & $13 \%$ \\
Regressão e Correlação & 4 & $7 \%$ \\
Análise Multivariada de & & \\
dados & 4 & $7 \%$ \\
Abordagem multicritério & 0 & $0 \%$ \\
Modelagem Matemática & 1 & $2 \%$ \\
\hline
\end{tabular}

Fonte: dados da pesquisa, 2017 
A classificação dos métodos estatísticos informados na Tabela 5 teve como base o referencial teórico dessa pesquisa, conforme classificação dos pesquisadores Dallanona, Nascimento e Hein (2010, p. 60) à luz dos autores Spiegel (1993), Maroco (2003) e L. J. Corrar, Paulo e Dias Filho (2007), V. DosSantos, Klann, Rausch (2010).

Conforme informado na Tabela 5, consideradas as duplicidades, ou seja, o mesmo artigo apresentam diversas técnicas estatísticas, $70 \%$ dos artigos utilizaram a estatística descritiva com distribuições de frequência, média, mediana, variância para os estudos em contabilidade gerencial, apresentando a maior representatividade nos assuntos pesquisados sobre o tema. Em seguida, o segundo método estatístico abordado foi a inferência estatística com $13 \%$ que usaram testes de hipóteses para amostras emparelhadas, testes paramétricos e não paramétricos e métodos quiquadrado. A terceira maior representatividade em relação ao total dos artigos com métodos estatísticos analisados foram à análise multivariada dos dados, com análise de grupamentos de dados, cluster, dendogramas nas abordagens da pesquisa em contabilidade gerencial e as medidas de Regressão e Correlação que ficaram com $7 \%$ da representatividade total, para este foram usados métodos de regressão logística e coeficientes de correlação como de Alfa de Cronbach, este último para estimar a confiabilidade do questionário aplicado na pesquisa realizada, como demonstrada na Figura 3.

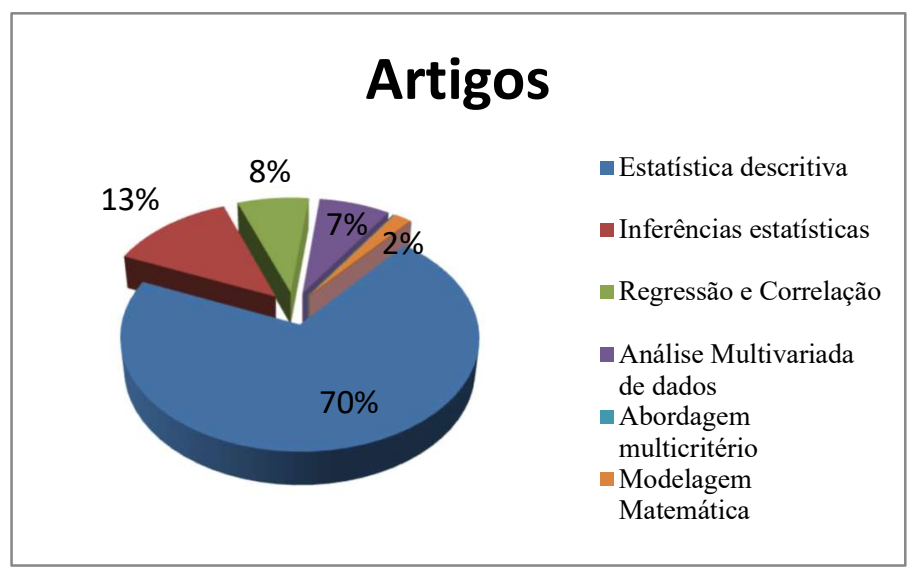

Figura 3: Percentual dos métodos estatísticos aplicados Fonte: Dados da pesquisa, 2017

\section{iii) Lei de Lokta para os artigos em Contabilidade Gerencial que Utilizaram Estatísticas}

$\mathrm{Na}$ Tabela 6 apresenta-se a produtividade dos autores segundo o critério da contagem completa das produções científicas com temas abordados em contabilidade gerencial que utilizaram a estatística em suas análises, relacionando a produtividade estimada pela Lei de Lokta com o Padrão dessa lei.

Tabela 6: Lei de Lokta para os artigos que utilizaram estatísticas

\begin{tabular}{cccccc}
\hline $\begin{array}{c}\text { Artigos } \\
\text { por autor }\end{array}$ & $\begin{array}{c}\text { Número de } \\
\text { autores } \\
\text { absoluto }\end{array}$ & $\begin{array}{c}\text { \% de autores } \\
\text { absolutos }\end{array}$ & $\begin{array}{c}\text { Número de } \\
\text { Autores } \\
\text { estimados Lei } \\
\text { de Lokta }\end{array}$ & $\begin{array}{c}\text { \%úmero de } \\
\text { autores } \\
\text { estimados Lei } \\
\text { de Lokta }\end{array}$ & $\begin{array}{c}\text { Padrão } \\
\text { Lokta }\end{array}$ \\
\hline 1 & 30 & $91 \%$ & 30 & $68 \%$ & $60,80 \%$ \\
\hline 2 & 1 & $3 \%$ & 8 & $17 \%$ & $15,20 \%$ \\
\hline 3 & 1 & $3 \%$ & 3 & $8 \%$ & $1,69 \%$ \\
\hline 5 & 0 & $0 \%$ & 2 & $4 \%$ & $0,11 \%$ \\
\hline TOTAL & 1 & $3 \%$ & 1 & $3 \%$ & $0,0 \%$ \\
\hline
\end{tabular}


$\mathrm{Na}$ Tabela 6, a frequência da produtividade dos autores de um artigo superou em 30,2 pontos percentuais, observando a permanência da grande concentração de autores que publicaram somente um artigo, indo ao encontro com a Lei de Lokta. Em relação à produtividade estimada através do quadrado inverso obtido por Lokta o percentual de produção superior foi menor em 23 pontos percentuais.

A média de trabalho por autor foi de 1,33, com variância de 60,5 e índice de dispersão medido igual a 7,78 artigos por autor.

Houve uma concentração menor de autores que produziram dois, três e cinco, conforme preconizado por esta lei, porém obtiveram discrepância da frequência percentual relativa se comparado à estimativa da Lei de Lokta. Além disso, convêm observar que a probabilidade estimada para publicação de cinco artigos foi satisfatória por apresentar em termos percentuais iguais ao percentual de frequência da produtividade dos autores absolutos.

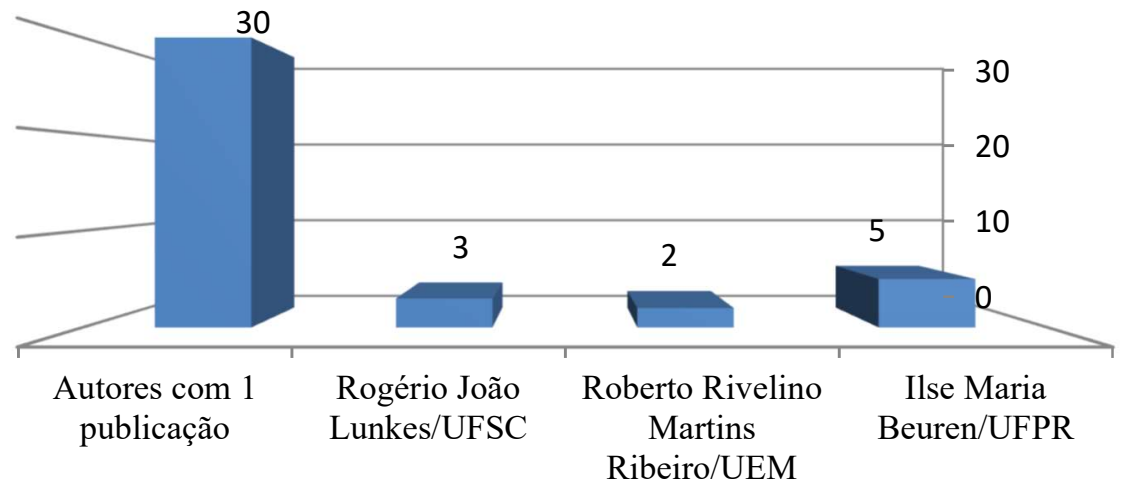

Figura 4: Autores com maior produção

Fonte: Dados da pesquisa, 2017

Agrupamento de dados e modelagem matemática, com cinco produções científicas, publicadas nas revistas, cujas principais são Revista de Educação e Pesquisa em Contabilidade (REPEC), Revista Eletrônica de Ciência Administrativa (RECADM) e Revista Contabilidade, Gestão e Governança (CGC). Em seguida, os pesquisadores Rogério João Lunkes com três artigos publicados usando a Estatística descritiva, Análise de homogeneidade de clusters e Análise multivariada dos dados, nas revistas da Universidade Estadual de Maringá (UEM), Revista da Universidade Federal de Santa Catarina e Advances in Scientific and Applied Accounting (ASAA Journal). Por fim, Rivelino Martins com dois artigos, utilizando os métodos estatísticos da Estatística descritiva e modelagem matemática nas revistas Revista Capital Científico-eletrônico (RCCe) e Revista Universidade Estadual de Maringá (UEM). E, 30 pesquisadores que publicaram somente um artigo de contabilidade gerencial usando estatística descritiva na maior parte dos estudos publicados.

\section{iv)Lei de Lokta para os artigos em Contabilidade Gerencial que mede a produtividade da categoria de método estatístico}

De acordo com a análise da Tabela 7, item ii da seção de análise dos dados, para a observação da produtividade dos autores que aplicaram os métodos estatísticos por meio da Lei de Lokta, será considerado a classificação estatística que tiveram a maior relevância no que se refere a representatividade nos trabalhos publicados em Contabilidade Gerencial que são estatísticas descritivas e inferência estatística. 
Tabela 7: Lokta sobre a Estatística Descritiva

\begin{tabular}{ccccc}
\hline $\begin{array}{c}\text { Artigos por } \\
\text { autor }\end{array}$ & $\begin{array}{c}\text { Número de } \\
\text { autores absoluto }\end{array}$ & $\begin{array}{c}\text { \% de autores } \\
\text { absolutos }\end{array}$ & $\begin{array}{c}\text { Número de Autores } \\
\text { estimados Lei de Lokta }\end{array}$ & $\begin{array}{c}\text { \% de autor } \\
\text { estimado pela Lei } \\
\text { de Lokta }\end{array}$ \\
\hline 1 & 31 & $84 \%$ & 31 & $73 \%$ \\
\hline 2 & 2 & $5 \%$ & 8 & $18 \%$ \\
\hline 5 & 4 & $11 \%$ & 1 & $5 \%$ \\
\hline $\mathbf{3 7}$ & $\mathbf{1}$ & $\mathbf{4 0}$ & $\mathbf{1}$ \\
\hline
\end{tabular}

Sob modelo de contagem completa foi calculado o teste qui-quadrado de homogeneidade da distribuição pelo programa $\mathrm{R}$, a fim de obter a hipótese nula em que há associação entre o número de produtores observados (Número de autores absoluto) com o número de produtores estimados pela Lei de Lokta. Ao aplicar o cálculo pelo Pearson no R, ao nível de 0,05 nível de significância, com 2,5 graus de liberdade, foi encontrado um qui-quadrado 5,2911 e p-value (valor crítico) de 0,07096, ou seja o p-value de 7,096 \% é maior que o nível de significância de 5\%, aceitando-se, portanto, a hipótese nula.

A Figura 5 abaixo informa a produtividade dos autores absoluta versus estimado pela Lei de Lokta que possuíram produção científica em contabilidade gerencial aplicado ao método da estatística descritiva. A produção média foi da ordem de 9,44 por autor, com um desvio padrão de 12,42.

A análise sob a Lei de Lokta também mostrou que a tendência dos números de autores por artigo continua diminuindo, em forma de um $\mathrm{J}$ que permite verificar pela representatividade gráfica abaixo obtida pela Figura 5 .

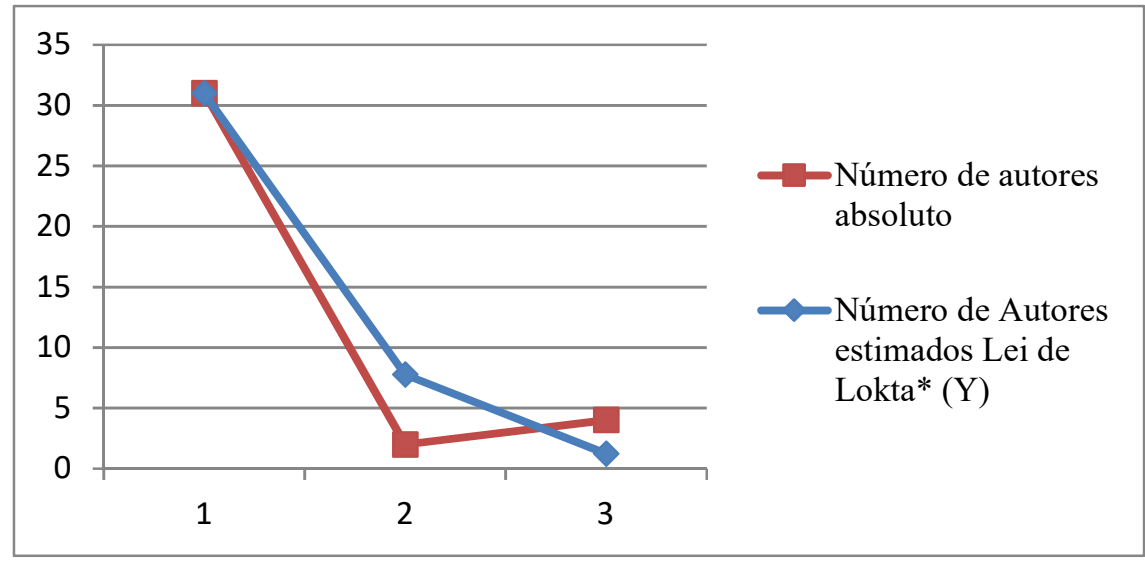

Figura 5: Produtividade sob a estatística descritiva Fonte: Dados da pesquisa, 2017

Tabela 8: Lokta sobre Inferência Estatística

\begin{tabular}{ccccc}
$\begin{array}{c}\text { Artigos por } \\
\text { autor }\end{array}$ & $\begin{array}{c}\text { Número de } \\
\text { autores absoluto }\end{array}$ & $\begin{array}{c}\text { \% de autores } \\
\text { absolutos }\end{array}$ & $\begin{array}{c}\text { Número de Autores } \\
\text { estimados Lei de } \\
\text { Lokta* (Y) }\end{array}$ & $\begin{array}{c}\text { \% de autores } \\
\text { pelo padrão Lei } \\
\text { de Lokta }\end{array}$ \\
\hline 1 & 7 & $100 \%$ & 7 & $60,8 \%$ \\
\hline
\end{tabular}

Fonte: Dados da pesquisa, 2017

A análise de Lokta para verificar a produtividade dos autores que utilizaram a inferência estatística não foi satisfatória e relevante, visto que somente obteve a quantidade de autores para produção de um artigo, não tendo informações suficientes para observação das premissas da Lei de Lokta. O ideal, segundo a literatura por Urbizagastegui (2008) e Filho (2008) seria a demonstração pela frequência maior de autores por produções científicas publicadas obtidas pelo quadrado inverso 
(Lokta). Nesse caso, observando graficamente na Figura 6, não houve a forma de J preconizado pela literatura de Lokta.

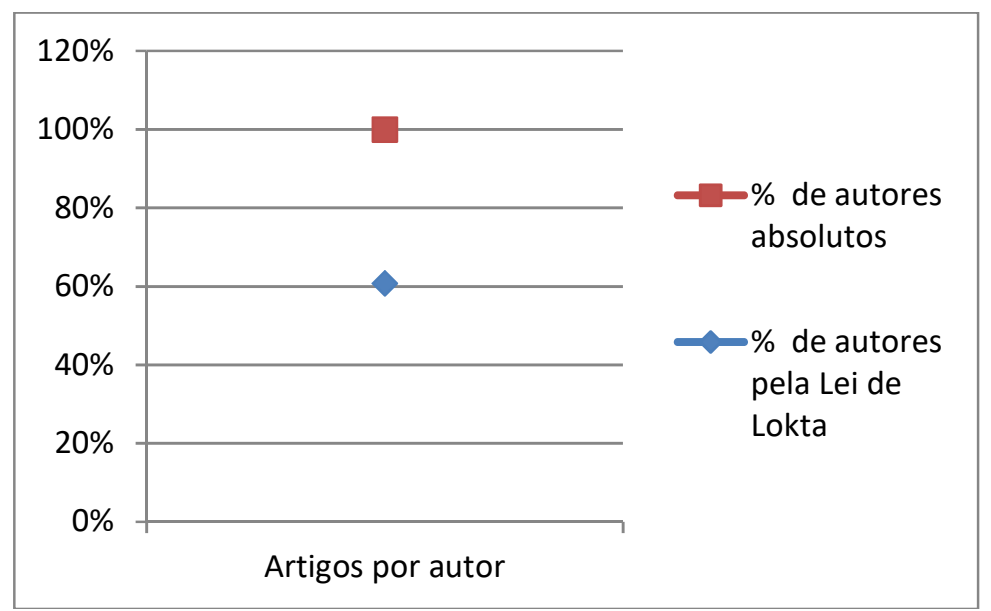

Figura 6: Produtividade da inferência estatística Fonte: Dados da pesquisa, 2017

\section{v) Comparação ao modelo de Poisson}

Devido aos resultados encontrados na análise dos métodos estatístico mais significativo aplicados na produção científica em Contabilidade Gerencial, nessa sessão foi abordada somente a produtividade em estatística descritiva para comparação no modelo de Poisson. Conforme ilustrado na Tabela 9, as produtividades estimadas pelo modelo de Poisson não conferem com as produtividades obtidas pela Lei de Lokta.

Tabela 9:Comparação ao modelo de Poisson Longnormal

\begin{tabular}{cccc}
$\begin{array}{c}\text { Número de } \\
\begin{array}{c}\text { Autores absoluto } \\
\text { estimado Lei } \\
\text { Lokta }\end{array}\end{array}$ & $\begin{array}{c}\text { Artigo } \\
\text { por } \\
\text { autor }\end{array}$ & $\begin{array}{c}\text { Número de } \\
\text { autores absoluto } \\
\text { pelo Poisson }\end{array}$ & $\begin{array}{c}\text { \% Artigo por } \\
\text { autor pelo } \\
\text { Poisson }\end{array}$ \\
31 & 1 & 12,4391244 & $33,62 \%$ \\
8 & 2 & 9,24529516 & $24,99 \%$ \\
1 & 5 & 0,50611875 & $1,37 \%$ \\
\hline
\end{tabular}

Fonte: Dados da pesquisa, 2017

Pelo modelo de Poisson os números de autores produziram somente um trabalho foram 12 comparados pela frequência relativa que tiveram um trabalho foi de 31 . A produção de 2 artigos ficou bem próxima a frequência da produtividade estimado por Lokta de 8 contra 9 de Poisson.

A Figura 7 mostra a curva de produtividade dos valores observados dos números de autores está bem próxima para forma de contagem na Lei de Lokta em forma de J, confirmado com o preconizado por Urbizagastegui (2008) em seu trabalho, porém a curva da produtividade pelo Poisson estimado pelo cálculo da média está bem divergente, conforme mencionado anteriormente. 


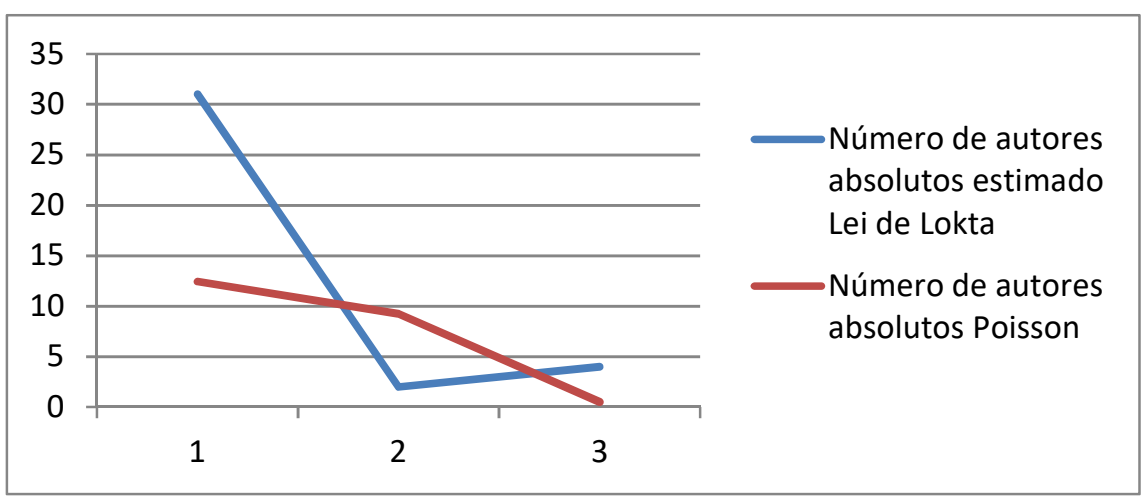

Figura 7: Número de autores por artigo versus autores estimado pelo Poisson Fonte: Dados da pesquisa, 2017

\section{CONSIDERAÇÕES FINAIS}

Esta pesquisa demonstra que, no período estudado de 2013-2016, a média total da produtividade dos autores que utilizaram os métodos estatísticos aos artigos publicados em temas abordados em Contabilidade Gerencial foi de 1,33 produções por autor e que a maior parte dessas produções aplicou a estatística descritiva em suas pesquisas.

Dentre as observações e análises realizadas das premissas da Lei de Lokta para os artigos em Contabilidade Gerencial que mede a produtividade da categoria de método estatístico, a produtividade média em estatística descritiva estimada pelo modelo probabilístico de Poisson foi de 1,3178. A curva de produtividade dos valores observados dos números de autores estimada por Lokta está bem próxima ao padrão dessa lei formando um J, conforme observado pelo Urbizagastegui (2008), porém a produtividade informada pela quantidade de trabalho por autor no modelo de Poisson está de encontro à produtividade pela Lei de Lokta.

Já na inferência estatística não foi satisfatória e relevante, visto que somente obteve a quantidade de autores para produção de um artigo, não tendo informações suficientes para observação das premissas da Lei de Lokta.

Segundo as análises realizadas, dentre os autores mais profícuos que utilizaram os métodos estatísticos, observados a classificação Dallanona, Nascimento e Hein (2010), nos estudos em Contabilidade Gerencial houve uma concentração de pequenos produtores de artigos em assuntos mais relevantes corroborando com os estudos da Lei de Lokta.

A distribuição da produtividade dos autores comparados e estimados pela Lei de Lokta pela forma completa de contagem, em análise da relevância dos métodos estatísticos aplicados sob a forma de estatística descritiva, através do teste de hipótese, concluiu-se que há associação entre o número de produtores observados (Número de autores absoluto) com o número de produtores estimados pela Lei de Lokta.

Essa pesquisa se limitou apenas no objetivo de mapear a utilização dos métodos estatísticos em contabilidade gerencial no período de 2013 a 2016 através da análise bibliométrica, não verificando por meio empírico a contribuição daqueles métodos nessa disciplina, o que contribui para futuras pesquisas com objetivos específicos de determinar aplicabilidade da estatística em Contabilidade Gerencial, evidenciando qual é o método mais eficaz que trará benefícios para a tomada de decisão.

\section{REFERÊNCIAS}

ALVARADO, R. U. (2007). A blibliometria: História, legitimação e estrutura. In: Para entender a ciência da informação. Lídia Maria Batista Brandão Toutain: Organizadora. Salvador: EDUFBA (pp. 185-217).

BLONKOSKI, P. R., BORTOLUZZI, S. C., \& ANTONELLI, R. A. (2014). Contabilidade Gerencial: Análise bibliométrica e sistêmica da literatura científica internacional. In: $14^{\circ}$ Congresso Controladoria e Contabilidade - USP. Novas Perspectivas na Pesquisa Contábil. São 
Paulo, 21 a 23 de julho de 2014. Disponível em: http://www.congressousp.fipecafi.org/anais/artigos142014/188.pdf

BOENTE D. R., MELO, C. L. L., TARSO, P., ARAUJO, A. O. (2006). Métodos de estimação de custos: Estudo de caso de uma empresa comercial com enfoque na análise de regressão. $I n: 13^{\circ}$ Congresso Brasileiro de Custos. Belo Horizonte. Anais. Belo Horizonte: Associação Brasileira de Custos. Disponível em: http://www.abcustos.org.br/texto/viewpublic?ID TEXTO=779

CHUEKE, G. V., AMATUCCI, M. (2015). O que é bibliometria? Uma introdução ao Fórum. Revista Eletrônica de Negócios Internacionais São Paulo, v.10, n. 2, pp. 1-5, mai./ago. Disponível em: http://internext.espm.br/index.php/internext/article/view/330/233

COELHO, C. U. F. (1999). Contabilidade Gerencial: Sistema de informação e controle. Revista Pensar Contábil do Conselho Regional de Contabilidade do Estado do Rio de Janeiro. Rio de Janeiro - RJ. n.3, ano II, mar.

CORRAR, L. J., \& THEÓPHILO, C. R. (2015). Pesquisa operacional para decisão em contabilidade e administração: Contabilometria. 2 Ed. São Paulo: Atlas.

CORRAR, L. J., PAULO, E., \& DIAS FILHO, J. M. (2007). Análise multivariada: Para os cursos de administração, ciências contábeis e economia. São Paulo: Atlas.

CREPALDI, S. A. Contabilidade gerencial. 4. Ed. São Paulo: Atlas, 2008.

DALlABONA, L. F., NASCIMENTO, S., \& HEIN, N. (2010). Métodos Estatísticos mais recorrentes nas dissertações do programa de pós-graduação em ciências contábeis da FURB. R. Cont. UFBA, Salvador-Ba, v. 4, n. 1, pp. 56-70, janeiro-abril. Disponível em: https://www.researchgate.net/publication/277210648_Metodos_Estatisticos_mais_Recorrentes_nas Dissertacoes do_Programa_de Posgraduacao_em_Ciencias_Contabeis_da_FURB

DASILVA, A. C., WANDERLEY, C. A. N. W., \& SANTOS, R. S. (in memorian). Utilização de ferramentas estatísticas em artigos sobre contabilidade financeira: Um estudo quantitativo em três congressos realizados no país. Revista Contemporânea de Contabilidade. UFSC, Florianópolis, ano 07, v.1, n. 14, p. 11-28, Jul./Dez., 2010- Publicado em 2011.

DASILVA, A. M. H. L., PONTES, A. V. V., \& VILLELA, S. M. (2010). Contabilidade gerencial aliada à mineração de dados. Revista Eletrônica da Faculdade Metodista Granbery- Curso de Administração, n. 9, Jul/dez. Disponível em: http://re.granbery.edu.br

DASILVA, M. C., CHACON, M. J. M., \& DOSSANTOS, J. (2005). O que é contabilometria? (What is "Contabilometria"?). Disponível em: www.atena.org.br/revista/ojs-2.2.38/index.php/pensarcontabil/article/viewFile/1533/1365

DESOUZA, M. A., LISBOA, L. P., \& ROCHA, W. (2003). Práticas de contabilidade gerencial adotadas por subsidiárias brasileiras de empresas multinacionais. Revista Contabilidade \& Finanças - USP, São Paulo, n. 32, pp. 40 - 57, maio/agosto.

DIEHL, C. A., DESOUZA, M. A., \& DOMINGOS, L. E. C. (2007). O uso da estatística descritiva na pesquisa em custos: Análise do XIV Congresso Brasileiro de Custos. ConTexto, Porto Alegre, v. 7, n. $12,2^{\circ}$ semestre.

DOSSANTOS, B. M., LEMES, P. T., SILVA, W. S., \& ALVES, A. F. (2014). A importância e o uso da estatística na área empresarial: uma pesquisa de campo com empresas do município de Elói Mendes - MG. Seminário de Excelência em Gestão e Tecnologia (SEGET), 31 de out. e 01 de nov.

FIGUEREDO, S. M. A., \& MOURA, H. J. M. (2000, agosto). A utilização dos métodos quantitativos pela contabilidade para otimização de receitas e racionalização de custos. VII Congresso Brasileiro de Custos - Recife, PE, Brasil. Disponível em: https://anaiscbc.emnuvens.com.br/anais/article/view/3006/3006 
FIGUEREDO, S. M. A., \& MOURA, H. J. M. (2001). A utilização dos Métodos Quantitativos pela Contabilidade. Revista Brasileira de Contabilidade, Brasília, ano 30, n.127, pp. 51-61, jan./fev.

FILHO, G. A. L. (2008). Padrőes de produtividade de autores em periódicos e congressos na área de contabilidade no Brasil: Um estudo bibliométrico. RAC, Curitiba, v. 12, n. 2, pp. 533 554, Abr./Jun.

IGNÁCIO, S. A. (2010). Importância da estatística para o processo de conhecimento e tomada de decisão. Revista Paranaense de Desenvolvimento, Curitiba, n.118. pp.175 - 192, jan./jun. Disponível em: http://www.ipardes.gov.br/biblioteca/docs/NT 06 importancia estatistica tomada decisao.pdf

IUDICÍBUS, S. (1982). Existirá a contabilometria? Revista Brasileira de Contabilidade. Brasília, $\mathrm{n}^{\mathrm{o}} 59$.

MARCONI, M. A., \& LAKATOS, E. M. (2002). Técnicas de pesquisa: Planejamento e execução de pesquisas, amostragens e técnicas de pesquisas, elaboração, análise e interpretação de dados. 5 Ed. São Paulo: Atlas.

MAROCO, J. Análise estatística com utilização do SPSS. 2 Ed. Lisboa: Edições Sílabo, 2003.

MONTEIRO, J. E. R., SILVA, G. I. S., Peter, M. G. A., \& MACHADO, M. V. V. (2011, outubro). Ética e Contabilidade: Estudo Bibliométrico das Publicações dos Anais do Enanpad na Primeira Década do Século XXI. Seminário em Administração - XIV SEMAD.

PEREIRA, A. T. Métodos quantitativos aplicados à contabilidade. Curitiba: InterSaberes, 2014.

PRODANOV, C. C., \& FREITAS, E. C. Metodologia do trabalho científico: Métodos e técnicas da pesquisa e do trabalho acadêmico. 2. Ed. Novo Hamburgo: Feevale, 2013.

REZENDE, L. F., \& CARMO, C. R. S. (2013). Métodos quantitativos aplicados à gestão de custos: Um estudo sobre ferramentas para a aplicação do custeamento variável e potencialização da sua utilidade gerencial. Universidade Federal de Uberlândia (FACIC-UFU) Brasil - Vol. XI, no 21, Jan-Jun.

RIBEIRO, R. R. M., \& ESPEJO, M. M. S. B. (2013). Evolução das pesquisas em contabilidade gerencial: Uma análise das opções temáticas em teses e dissertações no Brasil. Enfoque: Reflexão Contábil, v.32, $\quad$ n.1, pp. 49-63, jan- abril. Disponível em:http://www.periodicos.uem.br/ojs/index.php/Enfoque/article/view/18114/10978

SANTOS, V., KLANN, R. C., \& RAUSCH, R. B. (2010). Perfil das dissertações dos programas de pós graduação em ciências contábeis da FEA/USP e da FURB. In: Associação Nacional dos Programas de Pós-graduação em Ciências Contábeis, 4., 2010, Natal. Anais . . Natal: ANPCONT, 2010. CD-ROM. pp.1-15.

SELL, I. Utilização da regressão linear como ferramenta de decisão na gestão de custos. IX Congresso Internacional de Custos - Florianópolis, SC, Brasil - 28 a 30 de novembro, 2005.

SILVA, S. A. L., SANT`ANA, N. L. S., MENEZES, J. P. C. B., PAULA, J. S. de, \& CHAIN, C. P. Métodos e técnicas quantitativas em contabilidade e finanças: Uma análise sob a ótica do software R. Pensar Contábil, Rio de Janeiro, v. 19, n. 68, p. 37-46, jan./abr, 2017.

SPIEGEL, M. R. (1993). Estatística. 3 Ed. São Paulo: Makron Books.

URBIZAGASTEGUI, R. (2008). A produtividade dos autores sobre a Lei de Lotka. Ciência da Informação, v. 37, n. 2, p. 87-102, maio/ago.

VERGARA, S. C. (1998). Projetos e relatórios de pesquisa em administração. 2 Ed. São Paulo. Editora Atlas. 
WERNKE, R., \& BORNIA, A. C. (2001). A contabilidade gerencial e os métodos multicriteriais. Revista Contabilidade \& Finanças, v. 12, n. 25, São Paulo, Jan./Apr. Disponível em: http://www.scielo.br/scielo.php?script=sci_arttext\&pid=S1519-70772001000100004

\section{SOBRE OS AUTORES}

MARILÉA DA SILVA OLIVEIRA é mestranda em Ciências Contábeis/UERJ e graduada em Ciências Contábeis/UERJ.

E-mail: marileaoliveira@yahoo.com.br

ROBERTA DA SILVA LOPES é mestranda em Ciências Contábeis/UERJ, pós graduada em Implementação e Gestão na Educação à Distância/UFF, pós graduada em Matemática/UFF, graduada em Matemática/UFF e graduada em Ciências Contábeis/UNESA.

E-mail: lopesrobertadasilva@gmail.com

GUILHERME TEIXEIRA PORTUGAL é doutor em Ciências da Engenharia/UFRJ, mestre em Ciências Contábeis/UFRJ, pós graduado em Finanças/PUC-Rio e graduado em Administração de Empresas/PUC-Rio. Professor Adjunto da FAF/UERJ lecionando no programa stricto senso em Ciências Contábeis, sendo atualmente o coordenador do curso.

E-mail: teixteix@hotmail.com

JOSÉ FRANCISCO MOREIRA PESSANHA é doutor em Engenharia Elétrica/PUC-Rio, mestre em Engenharia Elétrica/UFRJ, graduado em Engenharia Elétrica/UERJ e em Estatística/ENCE. Professor do mestrado stricto senso em Ciências Contábeis/UERJ.

E-mail: professorjfmp@hotmail.com 